\title{
Condutas de puérperas imediatas frente a um suposto engasgo em bebês
}

\author{
Conducts of immediate puerperals in front of a suspended gagging in babies \\ Conductas de puerperales inmediatos frente un supuesto ahogo en bebés
}

Recebido: 05/08/2021 | Revisado: 11/08/2021 | Aceito: 17/08/2021 | Publicado: 20/08/2021

\author{
Antonio Filho Leal Lopes \\ ORCID: https://orcid.org/0000-0003-3669-0122 \\ Hospital Universitário Regional dos Campos Gerais, Brasil \\ E-mail: enfantfilholeal@gmail.com \\ Melina Lopes Lima \\ ORCID: https://orcid.org/0000-0002-8266-4040 \\ Hospital Universitário Regional dos Campos Gerais, Brasil \\ E-mail:enfmelina@gmail.com \\ Luciane Patrícia Andreani Cabral \\ ORCID: https://orcid.org/0000-0001-9424-7431 \\ Hospital Universitário Regional dos Campos Gerais, Brasil \\ E-mail: lucianepacabral@gmail.com \\ Everson Augusto Krum \\ ORCID: https://orcid.org/0000-0002-0342-6424 \\ Universidade Estadual de Ponta Grossa, Brasil \\ E-mail:everson.krum@uol.com.br \\ Cristina Berger Fadel \\ ORCID: https://orcid.org/0000-0002-7303-5429 \\ Universidade Estadual de Ponta Grossa, Brasil \\ E-mail: cbfadel@gmail.com
}

\begin{abstract}
Resumo
A obstrução de vias aéreas provocada por engasgo é um acidente grave e potencialmente fatal muito comum entre lactentes e crianças nos primeiros anos de vida, mas pode ocorrer em qualquer faixa etária. O objetivo deste estudo é investigar a conduta de puérperas internadas em um hospital de ensino frente a um suposto engasgo em bebês. A amostra foi constituída por puérperas imediatas internadas na maternidade de um hospital público de ensino. Foram coletados dados quantitativos e qualitativos de caracterização sociodemográfica, de saúde e de conhecimento sobre engasgo em bebês. Participaram 61 mulheres, sendo a maioria solteira, com ensino médio completo e renda familiar de até dois salários mínimos. Em relação ao treinamento sobre a manobra de desengasgo, 85,2\% nunca havia realizado e 95,1\% julgava importante. Das informações qualitativas foram extraídos três temas: autonomia do cuidado em saúde, transferência do cuidado em saúde e incapacidade, os quais deram origem a seis ideias centrais. Conclui-se que há um déficit importante de conhecimento e de conduta assertiva entre as puérperas acerca do tema, o que reforça a importância do investimento de equipes hospitalares na capacitação de mães e suas famílias no planejamento de alta.
\end{abstract}

Palavras-chave: Mães; Recém-nascido; Atitude frente à saúde; Engasgo.

\begin{abstract}
Airway obstruction caused by choking is a serious and potentially fatal accident that is very common among infants and children in their first years of life, but it can occur in any age group. The aim of this study is to investigate the behavior of postpartum women admitted to a teaching hospital when faced with supposed choking in babies. The sample consisted of immediate postpartum women admitted to the maternity ward of a public teaching hospital. Quantitative and qualitative data on sociodemographic, health and knowledge about choking in infants were collected. Sixty-one women participated, most of them single, with high school education and a family income of up to two minimum wages. Regarding the training on the degassing maneuver, $85.2 \%$ had never done it and $95.1 \%$ considered it important. Three themes were extracted from qualitative information: autonomy of health care, transfer of health care and disability, which gave rise to six central ideas. It is concluded that there is an important deficit of knowledge and assertive behavior among postpartum women on the subject, which reinforces the importance of investment by hospital teams in the training of mothers and their families in planning for discharge.
\end{abstract}

Keywords: Mothers; Newborn; Attitude of health; Choking.

\section{Resumen}

La obstrucción de las vías respiratorias causada por asfixia es un accidente grave y potencialmente fatal que es muy común entre bebés y niños en sus primeros años de vida, pero puede ocurrir en cualquier grupo de edad. El objetivo de este estudio es investigar el comportamiento de las puérperas ingresadas en un hospital universitario ante un supuesto 
atragantamiento en los bebés. La muestra estuvo constituida por mujeres postparto inmediato ingresadas en la sala de maternidad de un hospital universitario público. Se recogieron datos cuantitativos y cualitativos sobre aspectos sociodemográficos, de salud y conocimientos sobre asfixia en lactantes. Participaron 61 mujeres, la mayoría solteras, con estudios secundarios y un ingreso familiar de hasta dos salarios mínimos, en cuanto a la capacitación sobre la maniobra de desgasificación, el 85,2\% nunca la había realizado y el 95,1\% la consideraba importante. De la información cualitativa se extrajeron tres temas: autonomía de la asistencia sanitaria, transferencia de la asistencia sanitaria y discapacidad, que dieron lugar a seis ideas centrales. Se concluye que existe un importante déficit de conocimiento y comportamiento asertivo entre las puérperas sobre el tema, lo que refuerza la importancia de la inversión de los equipos hospitalarios en la formación de las madres y sus familias en la planificación del alta.

Palabras clave: Madres; Recién nacidos; Actitud hacia la salud; Ahogo.

\section{Introdução}

A impactação acidental de objetos no trato respiratório, conhecida como Obstrução das Vias Aéreas por Corpo Estranho - OVACE e habitualmente denominada como engasgo, é uma emergência potencialmente fatal e uma causa prevalente e evitável de parada cardiorrespiratória (Salih, Alfaki \& Alam-Elhuda, 2016; Kernell, DePaola, Maglione, Ahern, Penney, \& Addiss, 2018). Essa condição pode se apresentar como engasgo total, com obstrução completa das vias respiratórias ou engasgo leve, com as vias respiratórias parcialmente livres. Em ambas as situações o conhecimento sobre o tema e a tomada rápida de atitude tornamse essenciais para a prevenção de complicações à saúde.

O engasgo pode ser observado em indivíduos de qualquer faixa etária, entretanto, tende a ocorrer muito frequentemente na população infantil e na idosa. Na faixa etária pediátrica, a aspiração de corpo estranho (ACE) é uma causa importante de acidentes, especialmente entre lactentes e crianças nos primeiros anos vida (Rodrigues, Teixeira, Nascimento, Carvalho, Gonçalves, Almeida, \& Ribeiro. 2016; Rodríguez, Passali, Gregori, Chinski, Tiscornia, Botto \& Cuestas, 2012).

Cerca de $80 \%$ dos episódios de aspiração de corpo estranho pediátricos ocorre em crianças menores de três anos, com o pico de incidência entre um e dois anos de idade (CDC, 2019) e tendência de maior risco entre meninos (Sidell et al., 2013).O fato pode estar primariamente associado à maior autonomia, mobilidade e desenvolvimento de habilidades motoras finas nesta faixa etária, facilitando, por exemplo, a inserção de um pequeno objeto na boca; à falta de dentes molares e mastigação efetiva; e mecanismos de deglutição descoordenados ou imaturos (Ruiz, 2020; American Academy of Pediatrics, 2010).

Apesar da literatura apontar que o número total de mortes de menores de cinco anos diminuiu em todo o mundo (World Health Organization, 2019), a morte causada por ACE é a quinta causa mais comum de mortalidade por lesão não intencional nos Estados Unidos e a principal causa em crianças menores de um ano (Ruiz, 2020). No Brasil, de acordo com dados do DATASUS e considerando os anos de 2018 e 2019, a média de óbitos na faixa etária infantil devido à inalação e ingestão de alimentos e outros objetos que causam obstrução do trato respiratório foi de 130,5/ano (DATASUS, 2021).

Dentre os indivíduos muito jovens, o corpo estranho costuma ser citado como um alimento, um brinquedo, uma moeda ou uma bateria, mas pode ocorrer com qualquer outro objeto de pequenas dimensões (Duckett, Bartman \& Roten, 2021), embrenhados no organismo por meio do ouvido, nariz e vias aéreas.

A prevenção é um elemento chave para a diminuição da morbimortalidade associada à aspiração de corpo estranho na população pediátrica e deve ser tratada de forma abrangente. Programas educativos dirigidos a pais e cuidadores infantis, tanto na prevenção de hábitos que predispõem a acidentes nesta faixa etária, como no ensino de técnicas básicas de desobstrução de vias aéreas devem ser altamente incentivados (Rodrigues et al., 2016; Montana, Salerno, Feola, Asmundo, Di Nunno, Casella \& Di Mizio, 2020), tanto por gestores educacionais como por agentes de saúde pública. Desta forma, estratégias como a ampliação do rigor para concessão de produtos infantis, o desenvolvimento de campanhas massivas de educação pública e o incentivo à vigilância constante de cuidadores, uma vez que a visualização do engasgamento é informação mais importante para um diagnóstico precoce (Rodrigues et al., 2016), constituem-se práticas singulares para a qualidade de vida na primeira infância. 
Frente ao exposto, o estudo objetiva investigar a conduta de puérperas internadas em um hospital de ensino frente a um suposto engasgo em bebês.

\section{Metodologia}

Adotou-se o modelo de estudo exploratório descritivo, com abordagem quanti-qualitativa, o qual evidencia a complementaridade entre métodos qualitativos e quantitativos e a possibilidade de encaminhar estratégias de integração na prática da investigação em saúde e de identificar características e especificidades de cada abordagem (Serapioni, 2000). A amostra foi constituída pela totalidade de puérperas imediatas que estiveram internadas na maternidade de um hospital de ensino do interior do Paraná, Brasil, no período de outubro a dezembro de 2019 (n=61) e que concordaram em participar do estudo.

Para a coleta de dados foram realizadas entrevistas gravadas, com duração média de 10 minutos, e roteiro semidirigido aplicado por dois pesquisadores treinados. Para a caracterização dos sujeitos e aquisição de informações quantitativas foram coletadas as seguintes informações: idade; cor da pele; estado civil; escolaridade; profissão; estar trabalhando antes da gravidez; número de filhos; história de aborto ou de natimorto; número de consultas pré-natais; idade gestacional do início do pré-natal; tipo de parto; se já recebeu treinamento sobre a manobra de desengasgo em bebês, importância atribuída ao treinamento sobre a manobra de desengasgo em bebês e para qual número ligaria em caso de emergência relativa ao bebê.

Para a apreensão do conhecimento sobre engasgo em bebês (informações qualitativas) foi realizada a seguinte questão disparadora: "Caso seu bebê sofresse um engasgo, o que você como mãe faria?"

Os sujeitos foram abordados durante o seu período de internação hospitalar, em momento considerado propício. Após explicitação por pesquisadores treinados sobre os objetivos do estudo, meios e intermeios de coleta, análise e divulgação de resultados, os indivíduos, quando em acordo, de livre e espontânea vontade, assinaram termo de consentimento. Após a coleta de dados foi realizada uma capacitação sobre as medidas indicadas em primeiros socorros para desengasgo de bebês pela American Heart Association.

As variáveis de caracterização dos sujeitos foram analisadas através de estatística descritiva e o material produzido por meio da questão disparadora foi tabulado por pesquisador único e tratado de acordo com a técnica de análise do Discurso do Sujeito Coletivo (DSC), proposta por Lefèvre (2010, 2014). Foram obtidas estatísticas descritivas de todas as questões fechadas.

Este estudo atendeu as normas da Resolução 466/2012 do Conselho Nacional de Saúde e a Declaração de Helsinki e recebeu apreciação ética do Comitê de Ética em Pesquisa (parecer n 3.297.143, CAAE: 12201619.6.0000.0105).

\section{Resultados e Discussão}

A amostra final foi composta por 61 participantes, sendo que a maioria é solteira $(52,5 \%)$, com um único filho (41\%), tem o ensino médio completo $(49,2 \%)$, estava trabalhando antes de engravidar $(65,6 \%)$, com renda familiar de até um salário mínimo $(29,5 \%)$ ou entre um e dois salários mínimos $(29,5 \%)$ e idade média de 25 anos (17 +_40).

Sobre aspectos gerais de saúde, 19,7\% realizou sete consultas pré-natais, 29,5\% iniciou o pré-natal com oito semanas, $73,8 \%$ teve parto normal. Em relação ao treinamento sobre a manobra de desengasgo, 85,2\% nunca realizou e 95,1\% julga importante receber treinamento sobre esta manobra. Ao serem indagadas acerca do número telefônico para situação de emergência com seu bebê, $31,1 \%$ não soube responder; 3,4\% apontou um número inexistente; $42,6 \%$ citou o número do Serviço de Atendimento Móvel de Urgência - SAMU (192); 13,1\% citou o número do Corpo de Bombeiros (193) e 9,8\% referenciou a Polícia Militar (190).

Das informações qualitativas obtidas neste estudo foram extraídos três temas: 1) Autonomia do cuidado em saúde,2) Transferência do cuidado em saúde, 3) Incapacidade e seis ideias centrais: 'utilização de manobras e técnicas, com base em 
conhecimento adquirido'; 'utilização de manobras e técnicas, com base em instinto materno', 'atuação de acordo com o instinto materno e solicitação de auxílio'; 'busca por um serviço de urgência e emergência'; 'busca por ajuda inespecífica'; 'desenvolvimento de instabilidade emocional', as quais deram origem a seis DSC (Tabela 1).

Tabela 1. Descrição dos temas, síntese das idéias centrais e proporção de respostas obtidas da questão disparadora, segundo puérperas internadas em um hospital de ensino. Município de estudo, 2019 (n=61).

\begin{tabular}{|c|c|c|}
\hline TEMA & IDEAIS CENTRAIS & $\%$ \\
\hline \multirow{3}{*}{$\begin{array}{l}\text { AUTONOMIA DO CUIDADO } \\
\text { EM SAÚDE }\end{array}$} & $\begin{array}{l}\text { Utilização de manobras e técnicas, com base em conhecimento } \\
\text { adquirido }\end{array}$ & 38,2 \\
\hline & $\begin{array}{l}\text { Utilização de manobras e técnicas, com base em instinto } \\
\text { materno }\end{array}$ & 27,4 \\
\hline & $\begin{array}{l}\text { Atuação de acordo com o instinto materno e solicitação de } \\
\text { auxílio }\end{array}$ & 9,8 \\
\hline \multirow{2}{*}{$\begin{array}{l}\text { TRANSFERÊNCIA } \\
\text { CUIDADO EM SAÚDE }\end{array}$} & Busca por um serviço de urgência e emergência & 4,9 \\
\hline & Busca por ajuda inespecífica (pai, familiar, vizinho, etc) & 8,2 \\
\hline INCAPACIDADE & Desenvolvimento de instabilidade emocional & 11,5 \\
\hline
\end{tabular}

\section{Fonte: Autores (2020).}

\subsection{Tema 1: Autonomia do cuidado em saúde}

Assume-se, para a realização deste trabalho, que o processo de produção da autonomia em saúde de sujeitos individuais e coletivos está subordinado às suas capacidades de serem livres e autônomos para refletirem sobre as melhores escolhas em relação a sua própria saúde e de sua família (Brasil, 2015). A seguir, são expostas as opiniões críticas referentes a esse tema, dispostas em três idéias centrais, as quais deram origem aos discursos.

\subsubsection{Utilização de manobras e técnicas, com base em conhecimento adquirido}

Os discursos que seguem evidenciam que, apesar da demonstração de acesso à informação e de aquisição do conhecimento das mães sobre o tema engasgo em bebês, o mesmo ocorreu, por vezes, por meio de mídias sociais, sem especificação da autoria de profissionais da saúde.

DSC - “Ah eu virara ele de costinha, daí batia, não sei se duas ou três vez nas costinha dele, deixando livre o nariz e a boquinha. Depois virava, e fazia com o dedinho no peito dele também até ele voltar. E para apoiar, colocava ele na palma da mão, de costinhas, ou de bruços no colo. ”(P11, P24, P35, P50, P51) 
DSC - "Vi no jornal e em alguns vídeos que para desafogar tem também que segurar o queixo antes de virar de bruços e bater nas costas, mas sem colocar o dedo na boca. No vídeo mostrava como desengasgar, mas não lembro exatamente como é... só sei que ia virar de bruços e dar tapinhas nas costas. ” (P7, P22, P35, P60)

Apesar da relação entre profissional de saúde e paciente estar sendo transformada nos tempos atuais, passando de um relacionamento tradicional impositivo para um relacionamento baseado na informação sobre práticas e condutas de saúde (da Silva, Fenner, Vargas, Kruel \& Benedetti, 2021), por vezes, o conhecimento popular culturalmente arraigado ocupa ainda papel principal na tomada de decisão em saúde (Alvarenga, Soares, Sales \& Anjos-Filho, 2020).Autores apontam que o saber ou conhecimento popular, apesar de nem sempre coincidir com o saber técnico ou cientificamente comprovado, é realmente capaz de direcionar ações em saúde (Alvarenga., et al, 2020; da Silva et al., 2021), devendo então ser considerado por profissionais da saúde.

No caso específico do presente estudo, o resultado evidencia a necessidade de investimento em profissionais, de diversas áreas de atuação dentro da neonatologia e da saúde materno-infantil, para capacitar essas mães frente a possíveis emergências.

\subsubsection{Utilização de manobras e técnicas, com base em instinto materno}

Apesar de alguns autores considerarem que o instinto materno é um mito, uma vez que não se encontra nenhuma conduta universal e necessária para ser mãe (Ambrosini \& Stanghellini, 2012), optou-se pela utilização desse termo para percorrer a história das atitudes maternas frente à situação de engasgo.

DSC - "Iria me assustar né, mas o instinto materno incentiva a gente a tentar fazer algo... Ia desafogar, batia nas costinhas e erguia os bracinhos para cima, ou viraria ele de lado e daria uns tapinhas nas costas. Mas eu desengasgaria!” (P1, P12, $\mathrm{P} 47, \mathrm{P} 48)$

Culturalmente a mãe tende há estar mais tempo com os filhos e muitas das vezes é quem identifica as possíveis situações de perigo a que os bebês estão expostos, consequentemente detendo a tomada de decisão e atitudes imediatas (RESENDE, 2017).Como a autonomia em saúde pressupõe a capacidade de tomar decisões por si próprio, em função de crenças e valores pessoais (BUYTENDIJK, 2017), a informação profissional assertiva sobre como agir frente ao engasgo em bebês precisa ser disseminada, visto que pode ser o fator diferencial entre a vida e a morte da criança de pouca idade (Rocha, Feitosa, Carvalho, Dodt, Queiroz \& Chaves, 2019).

\subsubsection{Atuação de acordo com o instinto materno e solicitação de auxílio}

Esta ideia central traz a tomada de atitude materna baseada na junção de práticas pessoais e de apoio profissional como melhor alternativa de socorro ao bebê.

DSC - "Ah eu faria tudo para dar certo, aí se não desse, chamava o socorro dos bombeiros ou iria ligar para a pessoa mais próxima me ajudar. Tentaria primeiro fazer aquela manobra...ia virar de costinhas, e ia batendo, pra tentar desafogar, o mais rápido possível, ia tentando. Mas primeiro eu ia tentar desengasgar, mesmo sem saber, ia dar um jeito e aí chamava socorro." (P17, P50, P53, P58)

Apesar da maioria das mães ter afirmado que nunca realizou treinamento sobre manobra de desengasgo (85,2\%) e quase um terço delas não saber a quem recorrer em caso de engasgo $(31,1 \%)$, a atitude imediata adotada por conta própria ainda prevaleceu nesta ideia central. Estudos mostram que frente a situações de desespero, o enfrentamento ocorre ou por meio de perda da capacidade funcional (Mangili, 2017) ou da busca de recursos criativos (dos Santos Almeida, dos Santos Almeida, Luz, 
Alves, Bernardo, Ferreira \& de Santana, 2020), como sugere o discurso.

\subsection{Tema 2: Transferência do cuidado em saúde}

Tradicionalmente a transferência de cuidado (handoff ou handover) consiste na transferência da responsabilidade do cuidado do paciente, ou grupo de pacientes, para outra pessoa ou grupo de profissionais, de forma temporária ou definitiva (Alves $\&$ melo, 2019). No caso desse estudo, o termo será aplicado para as mães que alegaram que a primeira atitude frente a uma situação de engasgo de seu bebê seria buscar por ajuda externa.

Apesar da transferência de cuidado dever ser incentivada desde antes do nascimento do bebê e envolver quantidade substancial de educação do paciente e de promoção da saúde (Brasil, 2013) visando à sua autonomia na situação de engasgo envolvendo bebês, a primeira decisão a ser estimulada é a busca por um serviço de urgência e emergência especializado. $\mathrm{O}$ fato não diminui a importância de instrumentalizar mães e famílias sobre manobras de desengasgo em bebês.

Considerando ainda que a rede de atenção à saúde materno-infantil deve se estender para além da atenção básica e adentrar pontos de especialidades e unidades hospitalares (Rocha et al., 2019), cabe à equipe hospitalar fomentar a comunicação contínua e a transferência de informações médicas tornando paciente, familiares e cuidadores mais seguros para dar continuidade ao cuidado fora do ambiente hospitalar. A informação de como proceder em situação de risco ao bebê é uma relevante informação a ser destinada durante a atenção pré-natal.

Seguem os discursos formadores das ideias centrais relacionadas.

\subsubsection{Busca por um serviço de urgência e emergência}

DSC - "Eu tentaria procurar uma ajuda, ligar para um número de emergência. Ligaria pro SAMU, para os bombeiros... porque não tenho muita experiência. Iria primeiro ligar sim, porque na hora a gente dificilmente lembra o que fazer, e ainda mais que não tenho nenhum treinamento." (P7, P15, P33)

\subsubsection{Busca por ajuda inespecífica}

DSC - “Com certeza eu procuraria alguém que pudesse ajudar, porque eu não sei nada sobre isso não. Ah! Sei lá...eu ia sair e pedir ajuda, ia ligar pro pai do nenê e pedia pra ele correr atrás de alguém para ajudar.” (P9, P21, P23, P30)

\subsection{Tema 3: Incapacidade}

Foram incluídas nesta temática as mães que expuseram incapacidade absoluta de tomar decisões de saúde, com repercussões negativas em seu controle emocional. De acordo com a literatura, a incapacidade (disability) centra-se em coisas que as pessoas não podem fazer, incluindo o desempenho de papéis socialmente esperados (Sampaio \& Luz, 2009). Ainda, o que determina se uma pessoa é incapaz de resoluções no âmbito da saúde é a falta de compreensão do problema ou os riscos e benefícios significativos do tratamento proposto e suas alternativas.

Como anteriormente discutido, situações de desespero podem se desdobrar em perda de capacidades. Quando mães são expostas a situações graves envolvendo seus filhos, o modelo psíquico desenvolvido pode gerar um sentimento de fraqueza ou de impossibilidade de ação (Morais, Simões, Rodrigues, Batista, Lamy, Carvalho \& Ribeiro, 2017), causando ainda mais sofrimento e passividade frente à ausência de recursos profissionais e terapêuticos.

Um processo de capacitação contínuo em cuidado que envolva mães, familiares e cuidadores, com vistas a estimulá-los a agirem de forma consciente e segura frente aos desafios cotidianos, pode e deve ser desencadeado durante o período de cuidado pré-natal intra-hospitalar. Para a família, o contato com o neonatologista e o prestador de cuidados iniciais diminui a confusão, 
ansiedade e incerteza quanto ao agir em saúde (Morais et al., 2020).

Ressalta-se que no presente estudo $85,2 \%$ das puérperas afirmaram nunca ter realizado treinamento sobre prevenção de engasgo em bebês, apesar da maioria julgar importante $(95,1 \%)$.

\subsubsection{Desenvolvimento de instabilidade emocional}

DSC - "Não tenho nem ideia, porque eu me apavoro só de pensar! Acho que ia chorar, porque sou muito nervosa nessas situações, não ia mesmo saber o que fazer. Simplesmente ficaria desesperada.” (P2, P5, P18)

O puerpério é marcado por diversas alterações na vida da mulher e pela percepção de novos sentimentos como o medo, a frustração, a irritação e a sensação de afastamento da vida anterior, bem como as incertezas quanto a forma de cuidar e os potenciais perigos que podem surgir (Costa, Dalorsoletta, Warmling, Trevisan, Teixeira, Cavalheiri \& Perondi, 2020).

Neste contexto, é de fundamental importância trabalhar o desenvolvimento da confiança materna frente a situações de perigo, como o engasgo em bebês, visto que o desequilíbrio emocional ocasionado pelo estresse do puerpério pode amplificar a sua sensação de incapacidade.

\section{Conclusão}

Além das especificidades inerentes ao método qualitativo, como a limitação de generalização dos resultados em virtude do tamanho e da intencionalidade amostral, a escassez de pesquisas anteriores sobre o tema abordado foi considerada restritiva para o aprofundamento de alguns aspectos da discussão, evidenciando lacunas na literatura e a oportunidade de novas investigações. Nesse sentido, sugere-se a realização de novos estudos sobre o assunto, com um maior número amostral, com vistas a aprofundar o conhecimento sobre o tema.

Apesar disso, o presente estudo propiciou reflexões relevantes acerca da temática de desengasgo em bebês, as quais subsidiarão estratégias de educação em saúde sobre o tema.

O presente estudo possibilitou identificar um déficit importante de conhecimento e de conduta assertiva de puérperas frente a um suposto engasgo em bebês. As informações trazidas nesta pesquisa reforçam a importância do conhecimento voltado à prevenção de agravos relacionados ao engasgo, devido sua elevada incidência e letalidade expressiva quando o socorro não é prestado de forma adequada e em tempo hábil.

Diante deste contexto, trabalhar o desenvolvimento da confiança materna frente ao engasgo, através do ensinamento da manobra de desengasgo, considerando o saber popular bem como as crenças e valores pessoais, envolvendo todos os membros da família, parece ser de fundamental importância para qualificar a conduta das mães frente ao engasgo. Nesse sentido, o investimento de equipes hospitalares na capacitação de mães e demais membros da família no planejamento da alta hospitalar aumenta a segurança para a continuação do cuidado fora do ambiente hospitalar.

\section{Referências}

Alvarenga, P., Soares, Z. F., Sales, P. K. C., \& Anjos-Filho, N. C. (2020). Escolaridade materna e indicadores desenvolvimentais na criança: mediação do conhecimento materno sobre o desenvolvimento infantil. Psico, 51(1), e31622-e31622.]

Alves, M., \& Melo, C. L. (2019). Transferência de cuidado na perspectiva de profissionais de enfermagem de um pronto-socorro. Revista Mineira de Enfermagem, $23,1-9$.

Ambrosini, A., \& Stanghellini, G. (2012). Mitos da maternidade. O papel da cultura no desenvolvimento da depressão pós-parto. Annali dell'Istituto superiore di sanità , 48 , 277-286.

American Heart Association. Guidelines. Destaques das Diretrizes da American Heart Association. Atualização das diretrizes de RCP e ACE, 2015. AHA versão português, p.1-32, 2015. 
American Heart Association. Destaques das diretrizes de RCP e ACE de 2020 da American Heart Association. https://cpr.heart.org/en/resuscitation-science/cprand-ecc-guidelines

American Academy of Pediatrics. (2010). Policy Statement_-Prevention of Choking Among Children. Pediatrics, 125, 601-607.

Brasil (2019). Ministério da Saúde. Banco de dados do Sistema Único de Saúde-DATASUS. http://www2.datasus.gov.br/DATASUS/index.php?area=0205\&id=6938\&VObj=http://tabnet.datasus.gov.br/cgi/deftohtm.exe?sim/cnv/inf10

Brasil (2013). Ministério da Saúde. Secretaria de Atenção à Saúde. Departamento de Atenção Básica. Acolhimento à demanda espontânea: queixas mais comuns na Atenção Básica. Cadernos de $\quad$ Atenção https://bvsms.saude.gov.br/bvs/publicacoes/acolhimento_demanda_espontanea_queixas_comuns_cab28v2.pdf

Buytendijk, F. J. J. (2017). A gênese psicológica do espírito materno. Revista da Abordagem Gestáltica: Phenomenological Studies, 23(1), 111-120.

Centers for Disease Control and Prevention (CDC). Ten leading causes of death and injury. 2019. https://www.cdc.gov/injury/wisqars/LeadingCauses.html

Cheng, J., Liu, B., Farjat, A. E., \& Routh, J. (2017). The public health resource utilization impact of airway foreign bodies in children. International journal of pediatric otorhinolaryngology, $96,68-71$.

Costa, L. D., Dalorsoletta, K., Warmling, K. M., Trevisan, M. G., Géssica Tuani Teixeira, G. T., Cavalheiri, J. C., \& Perondi, A. R. (2020). Dificuldades maternas no cuidado domiciliar a recém-nascidos. Rev Rene, 21 , e44194.

Duckett, SA, Bartman, M., \& Roten, RA (2021). Asfixia. StatPearls Publishing LLC. https://www.ncbi.nlm.nih.gov/books/NBK499941/

Kernell, JW, DePaola, RV, Maglione, AM, Ahern, LN, Penney, NG, \& Addiss, DG (2018). Risco de eventos adversos de deglutição e engasgo durante a desparasitação em crianças em idade pré-escolar. PLoS negligenciou doenças tropicais, 12 (6), e0006578.

Lefevre, F., \& Lefevre, A. M. C. (2010). Pesquisa de representação social: um enfoque qualiquantitativo: metodologia do Discurso do Sujeito Coletivo. In Pesquisa de representação social: Um enfoque qualiquantitativo: metodologia do discurso do sujeito coletivo (pp. 224-224).

Lefevre, F., \& Lefevre, A. M. C. (2014). Discurso do sujeito coletivo: representações sociais e intervenções comunicativas. Texto \& Contexto-Enfermagem, 23(2), 502-507.

Mangili, V. R. (2017). Indicadores de depressão pós-parto, ansiedade e estresse maternos: influências sobre a interação mãe-bebê. Dissertação de mestrado, Universidade Estadual de São Paulo, Bauru, SP, Brasil.

Montana, A., Salerno, M., Feola, A., Asmundo, A., Di Nunno, N., Casella, F. \& Di Mizio, G. (2020). Risk management and recommendations for the prevention of fatal foreign body aspiration: Four cases aged 1.5 to 3 years and mini-review of the literature. International Journal of Environmental Research and Public Health, 17(13), 4700.

Morais, A. O. D. D. S., Simões, V. M. F., Rodrigues, L. D. S., Batista, R. F. L., Lamy, Z. C., Carvalho, C. A. D., \& Ribeiro, M. R. C. (2017). Sintomas depressivos e de ansiedade maternos e prejuízos na relação mãe/filho em uma coorte pré-natal: uma abordagem com modelagem de equações estruturais. Cadernos de Saúde Pública, 33, e00032016.

Resende, D. K. (2017). Maternidade: Uma construção histórica e social. Pretextos-Revista da Graduação em Psicologia da PUC Minas, 2(4), 175-191.

Rodrigues, M., Teixeira, J., Nascimento, P., Carvalho, S., Gonçalves, A., Almeida, J., \& Ribeiro, C. (2016). Aspiração de corpo estranho na criança: um perigo escondido. Nascer e Crescer, 25, 173-176.

Rodríguez, H., Passali, GC, Gregori, D., Chinski, A., Tiscornia, C., Botto, H., \& Cuestas, G. (2012). Tratamento de corpos estranhos nas vias aéreas e esôfago. Jornal internacional de otorrinolaringologia pediátrica, 76, S84-S91.

Ruiz, F E (2020). Airway foreign bodies in children. In G B. Mallory (ed), S B. Torrey (ed), UpToDate. https://www.uptodate.com/contents/airway-foreignbodies-in-children?search=choking\&source=search_result\&selectedTitle=1 150\&usage_type=default\&display_rank=1\#references

Rocha, G. M. N., Feitosa, M. R., de Carvalho, R. E. F. L., Dodt, R. C. M., de Oliveira Queiroz, M. V., \& Chaves, E. M. C. (2019). Dúvidas maternas na alta hospitalar do recém-nascido. Revista Univap, 25(49), 93-103.

Salih, A. M., Alfaki, M., \& Alam-Elhuda, D. M. (2016). Airway foreign bodies: A critical review for a common pediatric emergency. World journal of emergency medicine, $7(1), 5$.

Silva, L. G., Fenner, P. C., Vargas, C. L., Kruel, C. S., \& Benedetti, F. J. (2021). Validação de um questionário para puérperas sobre o conhecimento em aleitamento materno. Brazilian Journal of Health Review, 4(1), 2303-2315.

Santos Almeida, N., dos Santos Almeida, N., Luz, D. C. R. P., Alves, F. F., Bernardo, R. V., Ferreira, E. L., \& de Santana, W. J. (2020). Conhecimento de professores do Ensino Fundamental sobre primeiros socorros no interior do Ceará: artigo original. Research, Society and Development, 9(9), e903998027e903998027.

Sampaio, R. F., \& Luz, M. T. (2009). Funcionalidade e incapacidade humana: explorando o escopo da classificação internacional da Organização Mundial da Saúde. Cadernos de Saúde Pública, 25, 475-483.

Serapioni, M. (2000). Métodos qualitativos e quantitativos na pesquisa social em saúde: algumas estratégias para a integração. Ciência \& Saúde Coletiva, 5 (1),187-192. 
Research, Society and Development, v. 10, n. 10, e590101019133, 2021

(CC BY 4.0) | ISSN 2525-3409 | DOI: http://dx.doi.org/10.33448/rsd-v10i10.19133

Sidell, D. R., Kim, I. A., Coker, T. R., Moreno, C., \& Shapiro, N. L. (2013). Food choking hazards in children. International journal of pediatric otorhinolaryngology, 77(12), 1940-1946.

World Health Organization (WHO). Children: reducing mortality. 2019. https://www.who.int/newsroom/fact-sheets/detail/children-reducing-mortality 